\title{
Word-representability of face subdivisions of triangular grid graphs
}

\author{
Herman Z.Q. Chen ${ }^{a}$, Sergey Kitaev ${ }^{b}$, Brian Y. Sun ${ }^{c}$ \\ ${ }^{a, c}$ Center for Combinatorics, LPMC-TJKLC \\ Nankai University, Tianjin 300071, P. R. China \\ ${ }^{b}$ Department of Computer and Information Sciences, \\ University of Strathclyde, Glasgow, G1 1XH, UK \\ Email: ${ }^{a}$ zqchern@163.com, ${ }^{b}$ sergey.kitaev@cis.strath.ac.uk, \\ ${ }^{c}$ brian@mail.nankai.edu.cn
}

\begin{abstract}
A graph $G=(V, E)$ is word-representable if there exists a word $w$ over the alphabet $V$ such that letters $x$ and $y$ alternate in $w$ if and only if $(x, y) \in E$. A triangular grid graph is a subgraph of a tiling of the plane with equilateral triangles defined by a finite number of triangles, called cells. A face subdivision of a triangular grid graph is replacing some of its cells by plane copies of the complete graph $K_{4}$.

Inspired by a recent elegant result of Akrobotu et al., who classified wordrepresentable triangulations of grid graphs related to convex polyominoes, we characterize word-representable face subdivisions of triangular grid graphs. A key role in the characterization is played by smart orientations introduced by us in this paper. As a corollary to our main result, we obtain that any face subdivision of boundary triangles in the Sierpinski gasket graph is wordrepresentable.
\end{abstract}

Keywords: word-representability, semi-transitive orientation, face subdivision, triangular grid graphs, Sierpiński gasket graph

\section{Introduction}

Let $G=(V, E)$ be a simple (i.e. without loops and multiple edges) undirected graph with the vertex set $V$ and the edge set $E$. We say that $G$ is wordrepresentable if there exists a word $w$ over the alphabet $V$ such that letters $x$ and $y$ alternate in $w$ if and only if $(x, y) \in E$ for any $x \neq y$.

The notion of word-representable graphs has its roots in algebra, where a prototype of these graphs was used by Kitaev and Seif to study the growth of the free spectrum of the well-known Perkins semigroup [10]. 
Recently, a number of (fundamental) results on word-representable graphs were obtained in the literature; for example, see [1], [3], [5], [7], [9], [11], and [12]. In particular, Halldórsson et al. [7] have shown that a graph is wordrepresentable if and only if it admits a semi-transitive orientation (to be defined in Section 2), which, among other important corollaries, implies that all 3-colorable graphs are word-representable. The theory of word-representable graphs is the main subject of the upcoming book [8].

The triangular tiling graph $T^{\infty}$ (i.e., the two-dimensional triangular grid) is the Archimedean tiling $3^{6}$ introduced in [13] and [4]. By a triangular grid graph $G$ in this paper we mean a graph obtained from $T^{\infty}$ as follows. Specify a number of triangles, called cells, in $T^{\infty}$. The edges of $G$ are then all the edges surrounding the specified cells, while the vertices of $G$ are the endpoints of the edges (defined by intersecting lines in $T^{\infty}$ ). We say that the specified cells, along with any other cell whose all edges are from $G$, belong to $G$. Any triangular grid graph is 3-colorable, and thus it is word-representable [7]. We consider non-3-colorable graphs obtained from triangular grid graphs by applying the operation of face subdivision which is defined in the sequel.

Subdividing a cell of a triangular grid graph means subdividing it into three parts by placing a vertex in the center of the cell and making it adjacent to the three cell's vertices. A face subdivision of a triangular grid graph is obtained by subdividing a number of specified cells in $G$.

Recently, Akrobotu at el. [1] proved that a triangulation of the graph $G$ associated with a convex polyomino is word-representable if and only if $G$ is 3-colorable. Inspired by this elegant result, in the paper in hands, we characterized word-representable face subdivisions of triangular grid graphs.

The paper is organized as follows. In Section 2 some necessary definitions, notation and known results are given. In Section 3 we state and prove our main result (Theorem 3.1) saying that a face subdivision of a triangular grid graph is word-representable if and only if it has no interior cell subdivided. Theorem 3.1 is proved using the notion of a smart (semi-transitive) orientation introduced in this paper. Finally, in Section 4 we apply our main result to face subdivisions of triangular grid graphs having equilateral triangle shape and face subdivisions of the Sierpiński gasket graph.

\section{Definitions, notation, and known results}

Suppose that $w$ is a word and $x$ and $y$ are two distinct letters in $w$. We say that $x$ and $y$ alternate in $w$ if the deletion of all other letters from the word $w$ results in either $x y x y \cdots$ or $y x y x \cdots$. 
A graph $G=(V, E)$ is word-representable if there exists a word $w$ over the alphabet $V$ such that letters $x$ and $y$ alternate in $w$ if and only if $(x, y) \in E$ for each $x \neq y$. We say that $w$ represents $G$, and such a word $w$ is called a word-representant for $G$. For example, if $w=134231241$ then the subword induced by the letters 1 and 2 is 12121, hence letters 1 and 2 are alternating in $w$, and thus the respective nodes are connected in $G$. On the other hand, the letters 1 and 3 are not alternating in $w$, because removing all other letters we obtain 1331; thus, 1 and 3 are not connected in $G$. One can see that w represents a 4 -cycle labelled by 1, 2, 3 and 4 in counter-clockwise direction.

If each letter appears exactly $k$ times in a word-representant of a graph, the word is $k$-uniform and the graph is said to be $k$-word-representable. For example, the word $w^{\prime}=13423124$ is also a word-representant for the 4cycle mentioned above, so the graph is 2-word-representable. The following theorem establishes equivalence of the notions of word-representability and uniform word-representability.

Theorem 2.1 ([9]). A graph $G$ is word-representable if and only if there exists $k$ such that $G$ is $k$-word-representable.

The graph $A^{\prime \prime}$ in Figure 2.1 will play a special role in this paper. Nonword-representability of $A^{\prime \prime}$ follows from the argument showing non-wordrepresentability of the graph co- $\left(T_{2}\right)$ in [6]. Indeed, $A^{\prime \prime}$ differs from co- $\left(T_{2}\right)$ in [6] only by the absence of the edges $(5,6),(5,7)$ and $(6,7)$, which did not affect the proof of the theorem in [6]. Thus, we omit the proof of the following theorem.

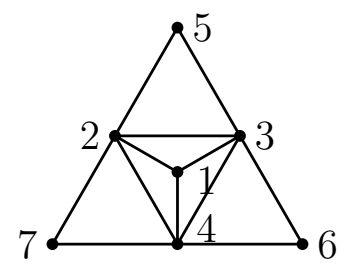

Figure 2.1: The graph $A^{\prime \prime}$.

Theorem 2.2. The graph $A^{\prime \prime}$ in Figure 2.1 is non-word-representable.

Next, we define key objects of our interest including semi-transitive orientations, triangular grid graphs and the Sierpinsski gasket graph. For graphtheoretic terminology not defined in this paper, the reader is referred to [2]. 


\subsection{Semi-transitive orientations}

A directed graph (digraph) is semi-transitive if it is acyclic, and for any directed path $v_{1} \rightarrow v_{2} \rightarrow \cdots \rightarrow v_{k}$ with $v_{i} \in V$ for all $i, 1 \leq i \leq k$, either

- there is no edge $v_{1} \rightarrow v_{k}$, or

- the edge $v_{1} \rightarrow v_{k}$ is present and there are edges $v_{i} \rightarrow v_{j}$ for all $1 \leq$ $i<j \leq k$. That is, in this case, the (acyclic) subgraph induced by the vertices $v_{1}, \ldots, v_{k}$ is transitive.

We call such an orientation a semi-transitive orientation.

We can alternatively define semi-transitive orientations in terms of induced subgraphs. A semi-cycle is the directed acyclic graph obtained by reversing the direction of one arc of a directed cycle. An acyclic digraph is a shortcut if it is induced by the vertices of a semi-cycle and contains a pair of non-adjacent vertices. Thus, a digraph on the vertex set $\left\{v_{1}, \ldots, v_{k}\right\}$ is a shortcut if it contains a directed path $v_{1} \rightarrow v_{2} \rightarrow \cdots \rightarrow v_{k}$, the arc $v_{1} \rightarrow v_{k}$, and it is missing an arc $v_{i} \rightarrow v_{j}$ for some $1 \leq i<j \leq k$; in particular, we must have $k \geq 4$, so that any shortcut is on at least four vertices. Slightly abusing the terminology, in this paper we refer to the arc $v_{1} \rightarrow v_{k}$ in the last definition as a shortcut (a more appropriate name for this would be shortcut arc). Figure 2.2 gives examples of shortcuts, where the edges $1 \rightarrow 4,2 \rightarrow 5$ and $3 \rightarrow 6$ are missing, and hence $1 \rightarrow 5,1 \rightarrow 6$ and $2 \rightarrow 6$ are shortcuts.

Thus, an orientation of a graph is semi-transitive if it is acyclic and contains no shortcuts.

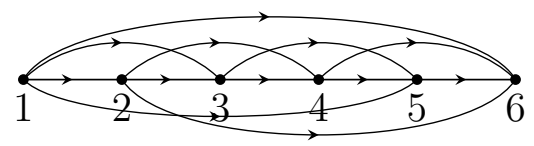

Figure 2.2: An example of a shortcut

Halldórsson et al. [7] proved the following theorem that characterizes word-representable graphs in terms of graph orientations.

Theorem 2.3 ([5]). A graph is word-representable if and only if it admits a semi-transitive orientation.

An immediate corollary to Theorem 2.3 is the following result.

Theorem 2.4 ([5]). 3-colorable graphs are word-representable. 


\subsection{Triangular grid graphs}

The infinite graph $T^{\infty}$ associated with the two-dimensional triangular grid (also known as the triangular tiling graph, see [13] and [4]) is a graph drawn in the plane with straight-line edges and defined as follows.

The vertices of $T^{\infty}$ are represented by a linear combination $x p+y q$ of the two vectors $p=(1,0)$ and $q=(1 / 2, \sqrt{3} / 2)$ with integers $x$ and $y$. Thus, we may identify the vertices of $T^{\infty}$ with pairs $(x, y)$ of integers, and thereby the vertices of $T^{\infty}$ are points with Cartesian coordinates $(x+y / 2, y \sqrt{3} / 2)$. Two vertices of $T$ are adjacent if and only if the Euclidean distance between them is equal to 1 (see Figure 2.3). A line $\ell$ containing an edge of $T^{\infty}$ is called a grid line. Note that the degree of any vertex of $T$ is equal to 6 . We refer to the triangular faces of $T^{\infty}$ as cells.

A triangular grid graph is a finite subgraph of $T^{\infty}$, which is formed by all edges bounding finitely many cells. Note that in our definition, a triangular grid graph does not have to be an induced subgraph of $T^{\infty}$. It is easy to see that $T^{\infty}$ is 3 -colorable, and thus any triangular grid graph is 3-colorable. Therefore, triangular grid graphs are word-representable by Theorem 2.4.

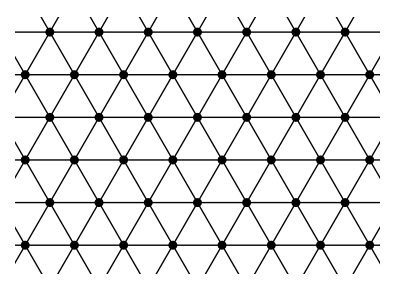

Figure 2.3: A fragment of the graph $T^{\infty}$.

The operation of face subdivision of a cell is putting a new vertex inside the cell and making it to be adjacent to every vertex of the cell. Equivalently, face subdivision of a cell is replacing the cell (which is the complete graph $K_{3}$ ) by a plane version of the complete graph $K_{4}$. A face subdivision of a set $S$ of cells of a triangular grid graph $G$ is a graph obtained from $G$ by subdividing each cell in $S$. The set $S$ of subdivided cells is called a subdivided set. For example, Figure 2.4 shows $K_{4}$, the face subdivision of a cell, and $A^{\prime}$, a face subdivision of $A$.

If a face subdivision of $G$ results in a word-representable graph, then the face subdivision is called a word-representable face subdivision. Also, we say that a word-representable face subdivision of a triangular grid graph $G$ is maximal if subdividing any other cell results in a non-word-representable graph.

An edge of a triangular grid graph $G$ shared with a cell in $T^{\infty}$ that does 


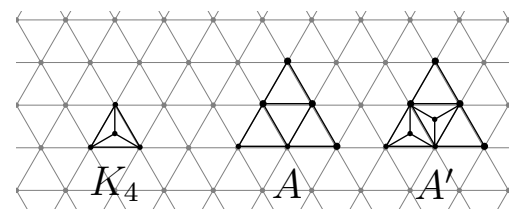

Figure 2.4: Examples of face subdivisions: $K_{4}$ is the face subdivision of a cell, and $A^{\prime}$ is a face subdivision of $A$.

not belong to $G$ is called a boundary edge. Recall that a cell belongs to $G$ if and only if all of its edges belong to $G$. A non-boundary edge belonging to $G$ is called an interior edge. A cell in $G$ that is incident to at least one boundary edge is called a boundary cell. A non-boundary cell in $G$ is called an interior cell. We denote by $v_{1}-v_{2}-v_{3}$ the cell whose vertices are labelled by $v_{1}, v_{2}, v_{3}$. The boundary edges in the graphs $H$ and $K$ in Figure 2.5 are in bold.

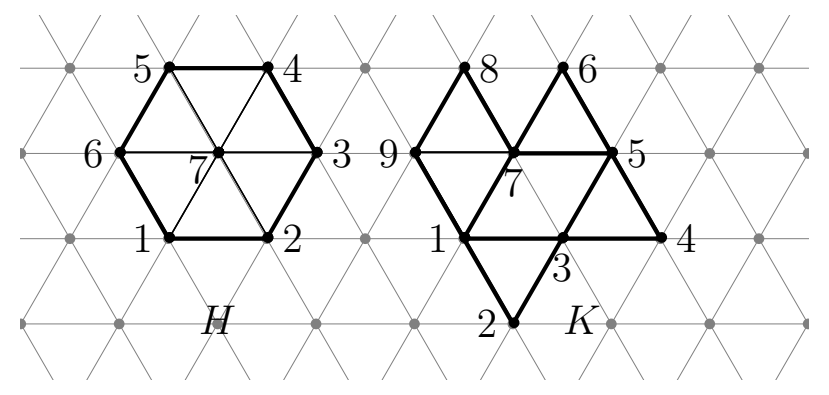

Figure 2.5: Graphs $H$ and $K$, where boundary edges are in bold.

A face subdivision of a triangular grid graph that involves face subdivision of just boundary cells is called a boundary face subdivision. A boundary edge parallel to the edge $(1,2)$ (resp., $(2,3),(3,4),(4,5),(5,6)$ and $(1,6))$ in the graph $H$ in Figure 2.5 and having the same layout of the boundary cell incident to it, is of type S (resp., SE, NE, N, NW and SW), which stands for "South" (resp., "South-East", "North-East", "North", "NorthWest" and "South-West"). For example, the boundary edges of the graph $K$ in Figure $2.5(1,2)$ and $(1,9)$ are of type SW, while the boundary edges $(3,5),(7,6)$ and $(8,9)$ are of type NW. The property set of a boundary cell is the set of types of boundary edges incident to the cell. For example, in the graph $K$ in Figure 2.5, the types of the cells 1-2-3 and 1-7-9 are $\{\mathrm{N}, \mathrm{SW}$, $\mathrm{SE}\}$ and $\{\mathrm{SW}, \mathrm{SE}\}$, respectively. 


\subsection{Sierpiński gasket graph}

The two-dimensional Sierpiński gasket graph, a lattice version of the Sierpiński gasket, also known as Sierpinski triangle, is one of the most studied selfsimilar fractal-like graphs. The construction of this graph, denoted by $S G(n)$ for initial stages is shown in Figure 2.6. At stage $n=0$, it is an equilateral triangle, i.e. a cell in $T^{\infty}$, while a stage $n+1$ giving $S G(n+1)$ is obtained by the juxtaposition of three graphs $S G(n)$ constructed on stage $n$. It is not difficult to see that $S G(n)$ is a triangular grid graph and it has $\frac{3}{2}\left(3^{n}+1\right)$ vertices and $3^{n+1}$ edges.
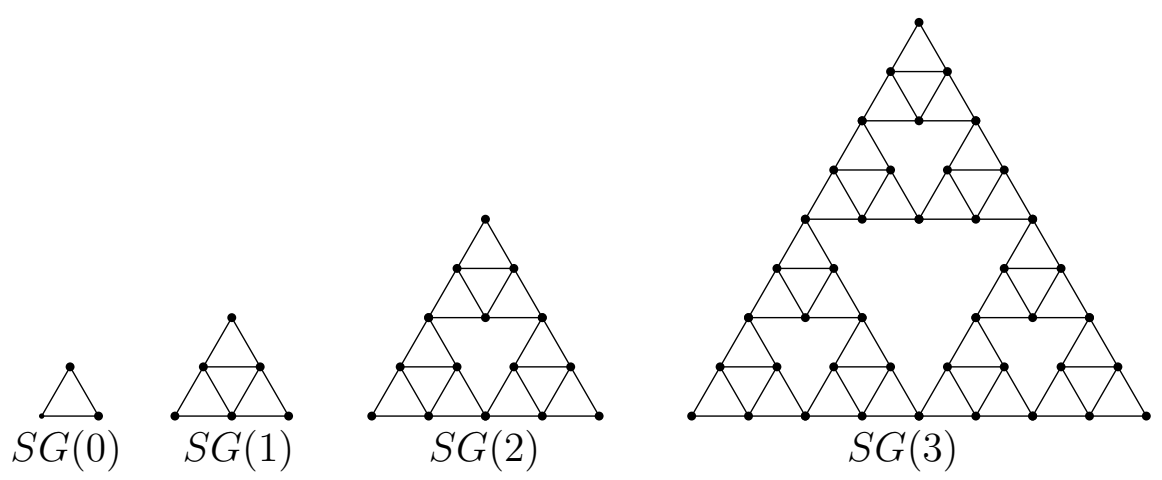

Figure 2.6: The first four stages corresponding to $n=0,1,2,3$ in construction of the two-dimensional Sierpiński gasket graph $S G(n)$.

\section{$3 \quad$ Face subdivisions of triangular grid graphs of general shape}

Clearly, the graph $A^{\prime \prime}$ in Figure 2.1 is isomorphic to a face subdivision of an interior cell in a triangular grid graph. Our main result is that subdividing all boundary (and no interior) cells of a triangular grid graph results in a word-representable graph.

Theorem 3.1. A face subdivision of a triangular grid graph $G$ is wordrepresentable if and only if it has no induced subgraph isomorphic to $A^{\prime \prime}$, that is, $G$ has no subdivided interior cell.

To prove Theorem 3.1, we will describe an orientation of a face subdivision of a triangular grid graph and then prove that this orientation is semi-transitive. First, define a correspondence between the types of boundary edges and the types of orientations of subdivided cells shown in Figure 3.7 

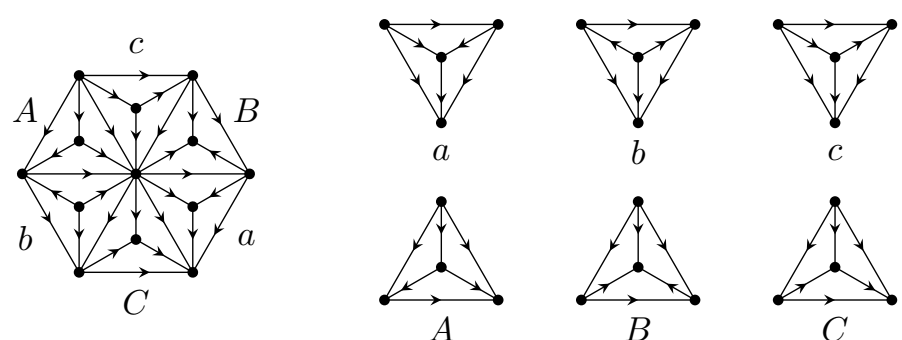

Figure 3.7: The semi-transitive orientation of the maximum face subdivision of the graph $H$, and six orientations of the subdivided cells.

as following: NW (respectively, NE, S, SE, SW, N) corresponds to $A$ (respectively, $B, C, a, b, c)$.

For a face subdivision $G^{\prime}$ of a triangular grid graph $G$ without subdivided interior cell, we direct the edges of $G^{\prime}$ as follows:

1. Direct all horizontal edges from left to right, and the edges forming $60^{\circ}, 90^{\circ}$, or $120^{\circ}$ with a horizontal line from top to bottom.

2. Direct the edges located inside subdivided cells consistently with an orientation shown in Figure 3.7 that corresponds to one of the types in the property set of the cell.

We refer to the arcs forming $0^{\circ}, 60^{\circ}$, or $120^{\circ}$ with a horizontal line as grid arcs. All other arcs are called by us inner arcs. If an orientation of a face subdivision of $G^{\prime}$ satisfies the two conditions above, then we say that the orientation is smart. Note that in general a smart orientation is not necessarily unique since a subdivided cell may have more than one boundary edge. An example of a smart orientation is the way we oriented the graph $H$ in Figure 3.7.
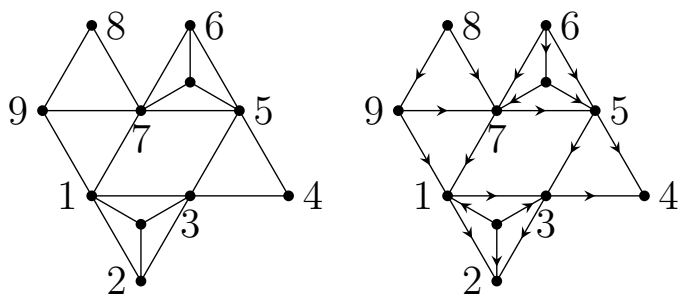

Figure 3.8: The face subdivision $K^{\prime}$ of the graph $K$ and one of its semitransitive orientations.

For another example, for the face subdivision of $K^{\prime}$ shown in Figure 3.8, the property sets of the subdivided cells $1-2-3$ and $5-6-7$ are, respectively, 
$\{\mathrm{SW}, \mathrm{SE}\}$ and $\{\mathrm{NW}, \mathrm{NE}\}$. Thus, referring to Figure 3.7, the orientations of edges for the cell $1-2-3$ can be chosen to be of type $a$ or $b$, while for the cell $5-6-7$ of type $A$ or $B$. In particular, the orientation shown in Figure 3.8 is smart, and this orientation can be checked by inspection to be semi-transitive.

Yet another example of a smart orientation is presented in Figure 3.9, where we also indicate types of orientations of cells used. In that figure we give a smart orientation for the graph obtained from subdividing all boundary cells in a triangular grid graph.
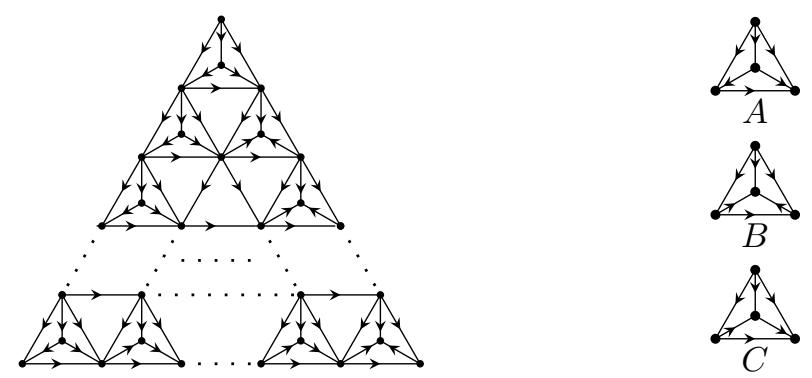

Figure 3.9: Semi-transitive orientation of $A_{n}$.

Note that a smart orientation may involve eight types of edges, that we call $a_{1}, a_{2}$, etc, $a_{8}$; see Figure 3.10.
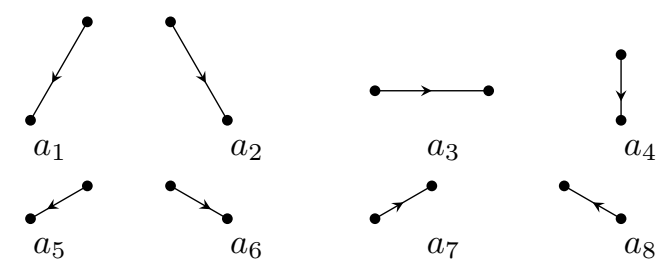

Figure 3.10: Eight types of oriented edges.

There are a number of properties that any smart orientation satisfies. Three of these properties, that are easy to see, are listed below. We will be using them, sometimes implicitly by considering fewer subcases, in the proof of Lemma 3.3:

- No directed path can connect a vertex on a horizontal line to another vertex to the left of it on the same line.

- No directed path can get from a horizontal line to a higher horizontal line. 
- The only situation in which a directed path can go down from a horizontal line and return back to it is when the upper inner arcs of type $c$ in Figure 3.7 are involved.

Lemma 3.2. No smart orientation of the boundary face subdivision of a triangular grid graph can have a directed cycle.

Proof. It is straightforward to see that no directed cycle is possible on just grid arcs, that is, when no inner arc is involved. Thus, if a directed cycle $C$ exists, then it must involve an inner arc.

Further, note that if $e_{1}$ is an inner arc in $C$, then there must exist an inner $\operatorname{arc} e_{2}$ that is located in the same cell as $e_{1}$, and $e_{1}$ and $e_{2}$ are next to each other in $C$. Without loss the generality, assume that $e_{1}=(x \rightarrow y)$ and $e_{2}=(y \rightarrow z)$. But then, looking at the six types of orientations of subdivided cells presented in Figure 3.7, we see that $e=(x \rightarrow y)$ is an arc in the oriented graph. Thus, we see that $C^{\prime}$ obtained from $C$ by removing $e_{1}$ and $e_{2}$ and including $e$ is still a directed cycle. Continuing in this manner, we can eliminate all inner arcs and show that there exists a directed cycle containing only grid arcs, which is impossible.

Thus, $C$ cannot exist, that is, any smart orientation is acyclic.

Lemma 3.3. No smart orientation of the boundary face subdivision of a triangular grid graph can contain a shortcut.

Proof. In a smart orientation, there are eight types of arcs shown in Figure 3.10, and we will prove that no $\operatorname{arc} e=(t \rightarrow h)$ can be a shortcut. While dealing with smart orientations, sometimes it is convenient to pay attention to coordinates $\left(x_{v}, y_{v}\right)$ of a vertex $v$ coming from the definition of $T^{\infty}$. The coordinates allow for any two vertices to determine which one of them is to the left of the other one and/or higher than the other one.

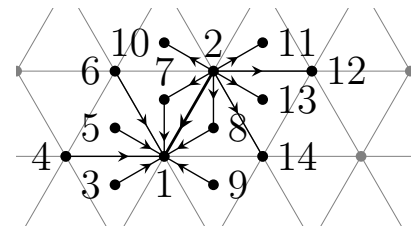

Figure 3.11: The case when the $\operatorname{arc} e=(2 \rightarrow 1)$ is of type $a_{1}$.

Case 1. Suppose that the $\operatorname{arc} e=(2 \rightarrow 1)$ is of type $a_{1}$, as shown in Figure 3.11. If $a_{1}$ is a shortcut, then there exists a directed path $P$ of length at least 3 from 2 to 1 (this path does not involve $e$ ). Consider the last vertex 
of $P$ that is on the horizontal line containing the vertex 2 . That vertex must actually be 2, otherwise we would obtain a contradiction with the semi-transitive orientation of the maximum subdivision of graph $H$ in Figure 3.7. So $P$ must start with $2 \rightarrow 7$ (in Figure 3.11 ), which is impossible by the following reason. The arc $2 \rightarrow 7$ is in orientation of type $a$ forcing $P$ be of length 2, contradiction. Thus, $e$ is not a shortcut in this case.

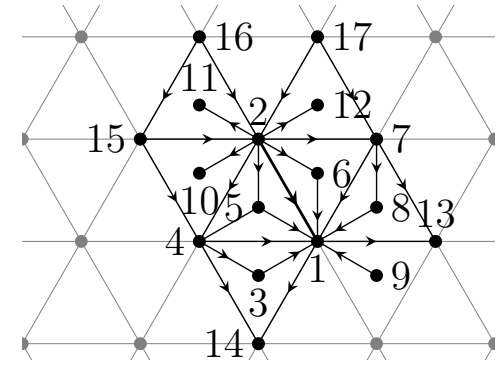

Figure 3.12: The case when the arc $e=(2 \rightarrow 1)$ is of type $a_{2}$.

Case 2. Suppose that the arc $e=(2 \rightarrow 1)$ is of type $a_{2}$, shown in Figure 3.12. If $a_{2}$ is a shortcut, then there exists a directed path $P$ of length at least 3 from 2 to 1 (this path does not involve $e$ ). Suppose that $P$ begins with $e^{\prime}$. Then $e^{\prime}=(2 \rightarrow s)$ can possibly be $2 \rightarrow 4,2 \rightarrow 5,2 \rightarrow 6$, $2 \rightarrow 7,2 \rightarrow 10,2 \rightarrow 11$, or $2 \rightarrow 12$.

Subcase 2.1 If $P$ begins with $2 \rightarrow 4$, according to the orientations, $P$ may be $2 \rightarrow 4 \rightarrow 5 \rightarrow 1,2 \rightarrow 4 \rightarrow 5 \rightarrow 3 \rightarrow 1$ or $2 \rightarrow 4 \rightarrow 3 \rightarrow 1$. Since the path $2 \rightarrow 4 \rightarrow 5 \rightarrow 1$ is semi-transitive, $P$ must be $2 \rightarrow 4 \rightarrow 5 \rightarrow 3 \rightarrow 1$ or $2 \rightarrow 4 \rightarrow 3 \rightarrow 1$. It forces the cell $1-4-14$ to have the orientation of type $c$, while $4 \rightarrow 1$ is not a boundary edge, contradicting the definition of a smart orientation.

Subcase 2.2 If $P$ begins with $2 \rightarrow 5$, then $P$ must be different from $2 \rightarrow 5 \rightarrow 1$. Moreover, since the path $2 \rightarrow 5 \rightarrow 4 \rightarrow 1$ is transitive, then $P$ must go through 3 to 1 (going to 14 is not an option since $P$ would never be able to return to the horizontal line the vertex 1 is on). But then the face subdivision of the cell 1-4-14 has the orientation of type $c$, while $4 \rightarrow 1$ is not a boundary edge, contradicting the definition of a smart orientation.

Subcase 2.3 If $P$ begins with $2 \rightarrow 6$, then the face subdivision of the cell 1-2-7 has the orientation of type $a$ or $c$. For the orientation of type $a, P$ is of length 2, contradiction. For the orientation of type $c$, since the path $2 \rightarrow 7 \rightarrow 6 \rightarrow 1$ is transitive, $P$ must go through 8 to 1 . Thus the face subdivision of the cell 1-7-13 has the orientation of 
type $A$, while $7 \rightarrow 1$ is not a boundary edge, contradicting with the definition of a smart orientation.

Subcase 2.4 If $P$ begins with $2 \rightarrow 7$, then $P$ must go through 8 to 1 . Similarly to the discussion in Subcase 2.2, it contradicts with the definition of a smart orientation.

Subcase 2.5 If $P$ begins with $2 \rightarrow 10, P$ must go through the $\operatorname{arcs} 4 \rightarrow 1$ or $4 \rightarrow 3$, while either of them indicates that $2 \rightarrow 4$ is not a boundary edge, contradicting the face subdivision of the cell 2-4-15.

Subcase 2.6 If $P$ begins with $2 \rightarrow 11$, it indicates that the orientation of the face subdivision of the cell $2-15-16$ is of type $B$. Thus the vertex 11 is a sink, contradiction.

Subcase 2.7 If $P$ begins with $2 \rightarrow 12$, the face subdivision of the cell 2-7-17 can possibly has the orientation of type $B$ or $C$. In the orientation of type $B$, the vertex 12 is a sink, contradiction. Thus the orientation is of type $C$, and $P$ must go through the arc $7 \rightarrow 1$ or $7 \rightarrow 8$, while either of them indicates that $7 \rightarrow 1$ is not a boundary edge, contradicting the face subdivision of the cell 2-7-17.

Thus, $e$ is not a shortcut in this case.

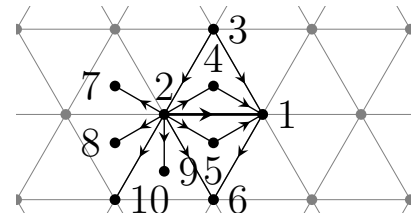

Figure 3.13: The case when the arc $e=(2 \rightarrow 1)$ is of type $a_{3}$.

Case 3. Suppose that the arc $e=(2 \rightarrow 1)$ is of type $a_{3}$, as shown in Figure 3.13. If $e$ is a shortcut, then there exists a directed path $P$ of length at least 3 from 2 to 1 (this path does not involve $e$ ). Suppose that $P$ begins with $e^{\prime}$. Then $e^{\prime}=(2 \rightarrow m)$ can potentially be $2 \rightarrow 4,2 \rightarrow 5$, $2 \rightarrow 6,2 \rightarrow 7,2 \rightarrow 8,2 \rightarrow 9$ or $2 \rightarrow 10$. However, $e^{\prime}$ cannot be $2 \rightarrow 8,2 \rightarrow 9$ or $2 \rightarrow 10$, because in each of these cases $P$ is forced to go through the vertex lying on the horizontal grid line below the vertex 1 , which is impossible by the properties of smart orientations listed above. Moreover, $e^{\prime}$ cannot be $2 \rightarrow 6$, since in this case $P$ cannot possibly return back to the vertex 1 since all the optional arcs are going down by the orientations of triangular grid graphs. Also, $e^{\prime}$ cannot be $2 \rightarrow 7$, since $2 \rightarrow 7$ must be an arc in subdiveded cell with orientation of type $B$ and therefore 7 must be a sink, contradiction. If $e^{\prime}$ is $2 \rightarrow 4$ or $2 \rightarrow 5$, then $P$ must be a path of length 2 , contradiction. Thus, $e$ is not a shortcut in this case. 


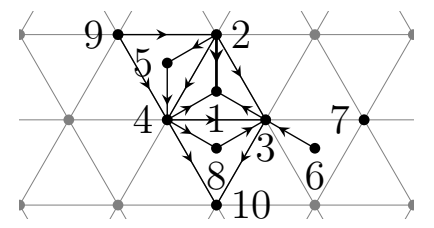

Figure 3.14: The case when the arc $e=(2 \rightarrow 1)$ is of type $a_{4}$.

Case 4. Suppose that the arc $e=2 \rightarrow 1$ is of type $a_{4}$, as shown in Figure 3.14. If $e$ is a shortcut, then there exists a directed path $P$ of length at least 3 from 2 to 1 (this path does not involve $e$ ). Then $P$ can possibly end with $3 \rightarrow 1$ or $4 \rightarrow 1$. There are two subcases:

Subcase 4.1 For ending with $3 \rightarrow 1$, the cell $2-3-4$ has the orientation $B$, so $2 \rightarrow 3$ is a boundary edge. If $4 \rightarrow 3$ lies on $P$, then $5 \rightarrow 4$ and $2 \rightarrow 5$ must lie on $P$ (since the path $2 \rightarrow 4 \rightarrow 1 \rightarrow 3$ is transitive and no directed path can connect a vertex on a horizontal line to another vertex to the left of it on the same line). Hence the subdivided cell $2-4-9$ has the orientation of type $a$ while $2 \rightarrow 4$ is not a boundary edge, contradicting the definition of a smart orientation. The arc $6 \rightarrow 3$ cannot lie on $P$, since existence of $6 \rightarrow 3$ implies that the vertex 6 is a source by the definition of a smart orientation. If $8 \rightarrow 3$ lies on $P$ then so does $4 \rightarrow 8$, hence the cell containing 8 has the orientation $c$, while $3 \rightarrow 4$ is not a boundary edge, contradicting the definition of a smart orientation.

Subcase 4.2 For ending with $4 \rightarrow 1$, since no directed path can connect a vertex on a horizontal line to another vertex to the left of it on the same line in a smart orientation, both $5 \rightarrow 4$ and $2 \rightarrow 5$ must lie on $P$, and hence the subdivided cell containing the vertex 5 has the orientation of type $a$, while $2 \rightarrow 4$ is not a boundary edge, contradicting the definition of a smart orientation.

Thus, $e$ is not a shortcut in this case.

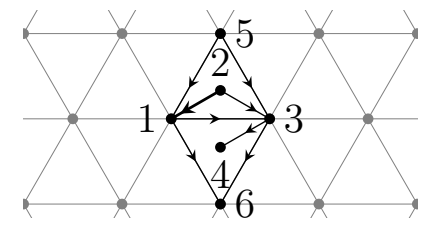

Figure 3.15: The case when the arc $e=(2 \rightarrow 1)$ is of type $a_{5}$.

Case 5. Suppose that the arc $e=(2 \rightarrow 1)$ is of type $a_{5}$, as shown in Figure 3.15. If $e$ is a shortcut, then there exists a directed path $P$ of length at least 
3 from 2 to 1 (this path does not involve $e$ ). Then $P$ must begin with the arc $2 \rightarrow 3$. However, no directed path can connect a vertex on a horizontal line to another vertex to the left of it on the same line, thus $e$ is not a shortcut.

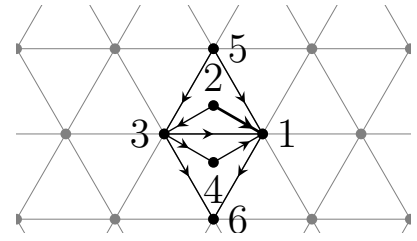

Figure 3.16: The case when the arc $e=(2 \rightarrow 1)$ is of type $a_{6}$.

Case 6. Suppose that the arc $e=(2 \rightarrow 1)$ is of type $a_{6}$, as shown in Figure 3.16. If $e$ is a shortcut, then there exists a directed path $P$ of length at least 3 from 2 to 1 (this path does not involve $e$ ). Then $P$ must begin with the arc $2 \rightarrow 3$. There are only two possibilities here: $P$ is either $2 \rightarrow 3 \rightarrow 1$, or $2 \rightarrow 3 \rightarrow 4 \rightarrow 1$. In the former case, we do not have a shortcut, while in the later case there is a contradiction with orientation of the cell $1-3-6$, since the arc $3 \rightarrow 1$ is not boundary. Thus, $e$ is not a shortcut in this case.

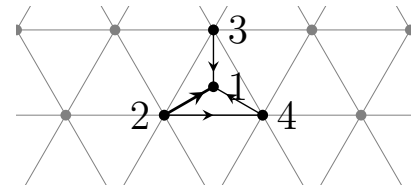

Figure 3.17: The case when the arc $e=(2 \rightarrow 1)$ is of type $a_{7}$.

Case 7. Suppose that the arc $e=(2 \rightarrow 1)$ is of type $a_{7}$, shown in Figure 3.17. If $e$ is a shortcut, then there exists a directed path $P$ of length at least 3 from 2 to 1 (this path does not involve $e$ ). Now $P$ can possibly end with $3 \rightarrow 1$ or $4 \rightarrow 1$. Since the vertex 3 lies on a horizontal line that is higher than the horizontal line the vertex 2 lies on, the case of $3 \rightarrow 1$ is impossible. On the other hand, showing that the case of $4 \rightarrow 1$ is impossible is similar to our considerations in Case 6.

Case 8. Suppose that the arc $e=(2 \rightarrow 1)$ is of type $a_{8}$, as shown in Figure 3.18. If $e$ is a shortcut, then there exists a directed path $P$ of length at least 3 from 2 to 1 (this path does not involve $e$ ). Now, $P$ can possibly end with $3 \rightarrow 1$ or $4 \rightarrow 1$. Both of these situations are impossible, first there exists no directed path from the vertex 2 to 3 by the property: 


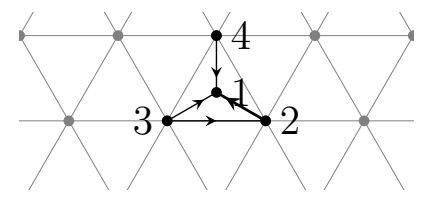

Figure 3.18: The case when the arc $e=(2 \rightarrow 1)$ is of type $a_{8}$.

no directed path can connect a vertex on a horizontal line to another vertex to the left of it on the same line; second, there exist no optional arcs whose arrow goes up for a directed path from the vertex 2 to 4 , i.e., the vertex 4 is not reachable from the vertex 2 by the property: no directed path can get from a horizontal line to a higher horizontal line in a smart orientations of a triangular grid graph.

By Lemmas 3.2 and 3.3, any smart orientation of the boundary face subdivision of a triangular grid graph is semi-transitive. Therefore, Theorem 3.1 is true by Theorem 2.3 .

\section{Concluding remarks}

In this paper we characterized word-representable face subdivisions of triangular grid graphs. We see our work as a step towards characterizing all word-representable planar graphs ${ }^{1}$. We conclude our paper with a discussion about an application of our main result to word-representability of face subdivisions of the celebrated Sierpiński gasket graph.

For the two-dimensional Sierpiński gasket graph $S G(n)$, by Theorem 3.1, we can obtain its maximum word-representable by subdividing all of its boundary cells. Figure 4.19 shows the maximum word-representable face subdivision of $S G(3)$ and one of its smart orientations.

$S G(n)$ can only have faces of degree $3 \cdot 2^{k}$, where $k=0,1, \ldots$, and the operation of face subdivision of a (triangular) cell can be generalized to face subdivision of other faces. One such generalization is inserting a new node inside a face and connecting it to all of the face's nodes. Another possible generalization is subdividing a face into three parts by connecting a newly added node to the three conner nodes of a face (note that each face being a $3 \cdot 2^{k}$-cycle, looks like a triangle, and the conner nodes are the vertices of such a triangle).

\footnotetext{
${ }^{1}$ Not all planar graphs are word-representable. The minimum non-word-representable (planar) graph is the wheel graph $W_{5}$ obtained by the face subdivision (in our sense) of the cycle graph $C_{5}$.
} 

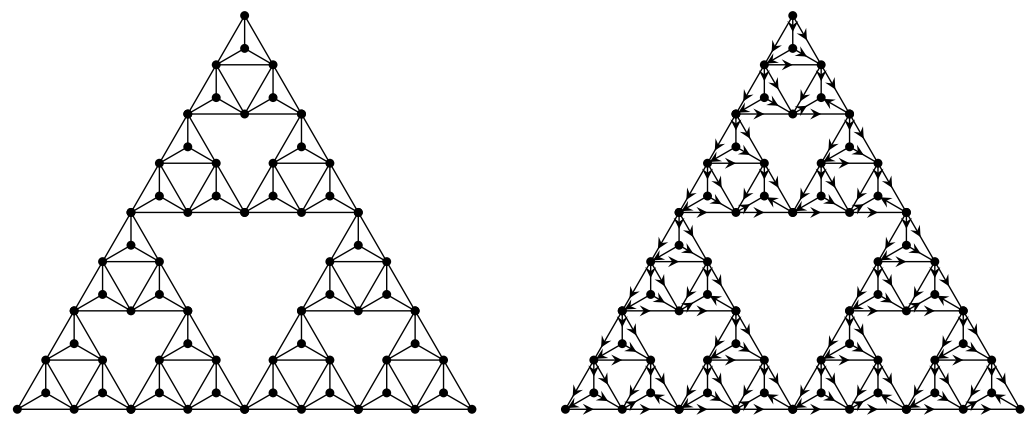

Figure 4.19: The maximum word-representable face subdivision of $S G(3)$ and one of its smart orientations.

We leave it as an open problem to study word-representability of the Sierpiński gasket graph when subdivision of faces of larger degrees is allowed. Studying this problem, again, will be a step towards characterizing wordrepresentable planar graphs.

\section{Acknowledgments}

The authors are grateful to the two anonymous referees for their useful suggestions. This work was supported by the 973 Project, the PCSIRT Project of the Ministry of Education and the National Science Foundation of China. Also, the second author is thankful to the Center for Combinatorics at Nankai University for its hospitality during the author's stay in November 2014.

\section{References}

[1] P. Akrobotu, S. Kitaev, Z. Masarova, On word-representability of polyomino triangulations, Siberian Adv. Math. 25 (2015) 1, 1-10.

[2] J.A. Bondy, U.S.R. Murty, Graph Theory with Applications, Macmillan, London, 1976, Elsevier, New York.

[3] A. Collins, S. Kitaev, V. V. Lozin, New results on word-representable graphs, Discrete Appl. Math. (2014), In Press.

[4] V. S. Gordon, Y. L. Orlovich, F. Werner, Hamiltonian properties of triangular grid graphs, Discrete Math. 308 (2008) 24, 6166-6188. 
[5] M. Halldórsson, S. Kitaev, A. Pyatkin, Alternation graphs, Lecture Notes in Comp. Sci. 6986 (2011) 191-202. Proceedings of the 37th International Workshop on Graph- Theoretic Concepts in Computer Science, WG 2011, Teplá Monastery, Czech Republic, June 21-24, 2011.

[6] M. Halldórsson, S. Kitaev, A. Pyatkin, Graphs capturing alternations in words. In Proceedings of the 14th international conference on Developments in language theory, DLT'10, pages 436-437, Berlin, Heidelberg, 2010. Springer-Verlag.

[7] M. Halldórsson, S. Kitaev, A. Pyatkin, Semi-transitive orientations and word-representable graphs, Discrete Appl. Math., to appear.

[8] S. Kitaev, V. Lozin, Words and graphs, Springer, to appear.

[9] S. Kitaev, A. Pyatkin, On representable graphs J. Autom. Lang. Comb. 13 (2008) 1, 45-54.

[10] S. Kitaev, S. Seif, Word Problem of the Perkins Semigroup via Directed Acyclic Graphs, Order 25 (2008) 3, 177-194.

[11] S. Kitaev, P. Salimov, C. Severs, H. Úlfarsson, On the representability of line graphs Lecture Notes in Computer Science 6795 (2011), 478479 Proc. 15th Conf. on Developments in Language Theory, DLT 2011, University of Milano-Bicocca, Milan, Italy, July 19-22, 2011.

[12] S. Kitaev, P. Salimov, C. Severs, H. Úlfarsson, Word-representability and line graphs, Open J. Discr. Math. 1 (2011) 2, 96-101.

[13] D. Ren, J. R Reay, The boundary characteristic and Pick's theorem in the Archimedean planar tilings, J. Combin. Theory, Series A 44 (1987) $1,110-119$. 